\title{
Autonomous Recombination and Self-healing of Fracture in Piezoelectric Organic Crystals: Scopes in Crystal Engineering
}

\author{
Surojit Bhunia, C. Malla Reddy,* \\ Department of Chemical Sciences, Indian Institute of Science Education and Research (IISER) Kolkata 741246, \\ Nadia, West Bengal (India) \\ cmallareddy@gmail.com
}

Stress-accumulated electrical charge to close wounds in living tissue, yet to-date this piezoelectric effect has not been realised in selfrepairing synthetic materials which are typically soft amorphous materials requiring external stimuli, prolonged physical contact and long healing times (often $>24 \mathrm{~h}$ ). Here we overcome many of these challenges using piezoelectric organic crystals, which upon mechanical fracture, instantly recombine without any external direction, autonomously self-healing in milliseconds with remarkable crystallographic precision (Figure 1). Atomic-resolution structural studies reveal that a 3D hydrogen bonding network, with ability to store stress, facilitates generation of stress-induced electrical charges on the fractured crystals, creating an electrostatically-driven precise recombination of the pieces via a diffusionless instant self-healing, as supported by spatially-resolved birefringence experiments. Perfect, instant self-healing creates new opportunities for deployment of molecular crystals using crystal engineering principles in robust miniaturised devices, and may also spur development of new molecular level repair mechanisms in complex biomaterials [1].

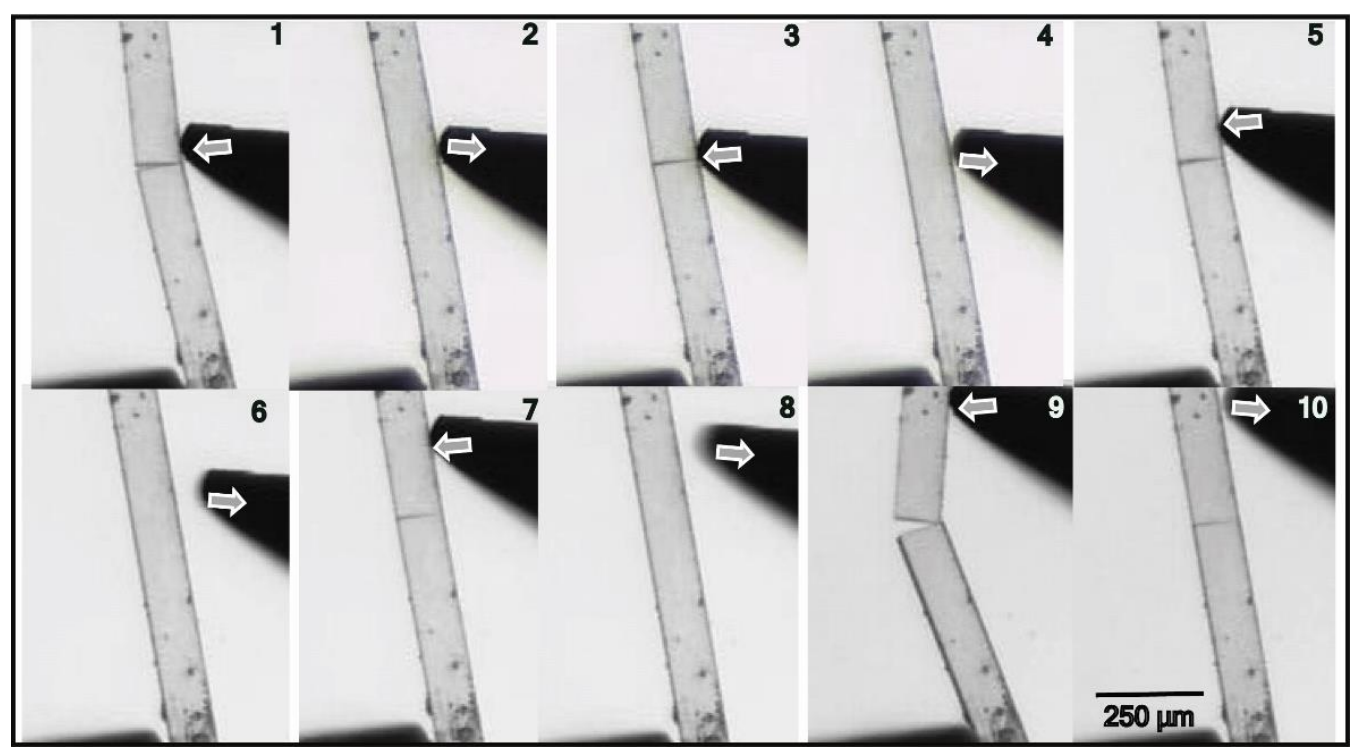

Figure 1. Self-healing cycles in the single crystal of bipyrazole derivative.

[1] Bhunia S, Chandel, S., Karan, SK., Dey, S., Tiwari, A., Das, S., Kumar, N., Chowdhury, R., Mondal, S., Ghosh, I., Mondal, A., Khatua, BB., Ghosh, N., Reddy, C. M. Autonomous self-repair in piezoelectric molecular crystals. (2021) Science. 373 , 321-327

Keywords: Self-healing in Piezoelectric, Crystal Engineering, Mechanical Property, Fracture Mechanics 